\title{
Semantic consistency of actions influences young children's word learning
}

\author{
Sarah F. V. Eiteljoerge ${ }^{1 *}$ ｜ Maurits Adam² | Birgit \\ Elsner $^{2}$ | Nivedita Mani ${ }^{1}$
}

${ }^{1}$ Psychology of Language, University of Goettingen, Goettingen, Germany

${ }^{2}$ Developmental Psychology, University of Potsdam, Potsdam, Germany

\section{Correspondence}

Sarah Eiteljoerge, Psychology of Language, University of Goettingen, Gosslerstr. 14, 37073 Goettingen, Germany Email: sarah.eiteljoerge@psych.unigoettingen.com

Funding information

This study was funded by the Deutsche Forschungsgemeinschaft (German Research Foundation), research unit "Crossing the borders: The interplay of language, cognition, and the brain in early human development" (Project: FOR 2253,

Grants: EL 253/7-1).
Communication with young children is often multimodal in nature, involving, for example, language and actions. This multimodal input supports language learning when it highlights the connection of word and object. But multimodal input can also guide the child's attention away from the language input, and thus, exacerbate learning. In the current study, we therefore examined the influence of semantic consistency of actions on early word learning. Children (18 months, 30 months, 36 - 48 months) and adults were presented with two novel objects and their novel labels while different actions were performed on these objects, such that the pairing of actions and objects was either consistent (Consistent group) or varied across trials (Inconsistent group). At test, participants saw both objects and heard one of the labels to examine participants' target looking upon hearing its label. Only 3- to 4-year-olds and adults learned word-object associations with the children benefiting from consistent and adults from the inconsistent action presentations. Thus, consistency in the multimodal input facilitated word learning in early childhood. In terms of a dynamic systems account of word learning, our study shows how multimodal learning settings interact with the child's perceptual abilities, and how the interaction can therefore shape the learning experience.

KEYWORDS 
word learning, actions, consistency, variability, cross-domain influences

\section{1 | INTRODUCTION}

Infants grow up in a multimodal world, where language input is typically embedded in a contextual framework. For example, this input can be provided with concurrently presented gestures like pointing and actions like the hopping of a rabbit. Indeed, words are often accompanied by actions (Matatyaho-Bullaro et al., 2014), and actions are often accompanied by words (e.g., Elsner and Pfeifer, 2012; Southgate et al., 2009). For preverbal infants, temporal alignment of parental language and actions in naming events is around $76 \%$, underscoring the co-occurrence of speech and action in early communication with infants (Gogate et al., 2000). This rich environment provides infants with the opportunity to experience and learn from different sources and senses but also requires the learner to play an active part in shaping the learning experience, filtering out what is relevant for her. How does a child determine what to focus on when confronted with simultaneous speech and action? Does information from one domain interfere with the processing in the other domain, or does cross-domain information foster processing on another domain? We examined these questions in the current study by investigating the role of semantic consistency of concurrently presented actions on word learning: in a word learning task, two groups of 18-, 30-month-old and 3- to 4-year-old children and adults saw two objects and heard their labels while the objects moved in either the same (consistent group) or in a varied (inconsistent group) manner across successive presentations of the word-object association. At test, participants' learning of the word-object associations were tested to see whether action consistency had an effect on their word learning.

The literature on early speech perception and word learning documents the impressive pace and flexibility with which infants acquire their native language, typically understanding and producing their first words even before their first birthday (e.g., Benedict, 1979; Bergelson and Swingley, 2012). Infant action learning proceeds at a similar pace. At 3 months already, infants can represent goal-based actions (Sommerville et al., 2005), and at least by 9 months they seem to understand the implications of actions (Behne et al., 2005). Also at this age, they have been shown to use the statistical probabilities of particular action units co-occurring with one another to segment action sequences (Stahl et al., 2014). Thus, already at 1 year of age, infants show impressive language and action processing skills.

Further, words and actions often come together: Actions caregiver speech in around $76 \%$ of naming events during year 1 (Gogate et al., 2000) and have already been shown to accompany 2-year-old children's speech (Nicoladis et al., 1999). Much work by Gogate and colleagues has investigated the role of mothers' action presentations during semistructured free play. They found that mothers favor certain action types in temporal synchrony with language in communicative interactions with their 6- to 8-month-olds (Matatyaho and Gogate, 2008). With time, the temporal synchrony of actions and words in maternal communication with infants reduces, adapting to the child's developmental needs and their reduced reliance on synchronicity in multimodal interactions (Gogate et al., 2000).

Young infants seem to benefit from multimodal input when, for example, they were presented with novel wordobject associations in semistructured play with their caregivers (Matatyaho and Gogate, 2008). In these studies, the concurrent presentation of actions and words or syllables helped 6- to 8-month-olds to learn the word-object mappings, as long as actions and words were temporally synchronous and infants attended to the presentation of multimodal input (e.g., Gogate et al., 2006). Similarly, in experimentally controlled habituation paradigms, 8-month-olds were better able to learn word-object mappings when the objects were presented with temporally synchronous actions, with some actions being favored compared to others (e.g., shaking compared to sideways, Matatyaho-Bullaro et al., 2014). Indeed, Werker et al. (1998) report that 14-month-olds learned the associations between words and their referents only when 
these objects were moving, but not when they were stationary. These results suggest that concurrently presented actions foster word learning in young children, potentially due to such multimodal content highlighting the connection between the word and the object. In contrast, Puccini and Liszkowski (2012) find that 15-month-olds learned words for objects, but not when they were accompanied by a referential action. Like the study by Matatyaho-Bullaro et al. (2014), these results suggest that the nature of the concurrently presented actions can influence young children's word learning.

Likewise, language input can influence children's interpretation of action demonstrations. For example, language can help structure action sequences (i.e., acoustic packaging, Brand and Tapscott, 2007; Meyer et al., 2011) and modulate children's representation of goal-directed actions (Elsner and Pfeifer, 2012), by highlighting the relevance of an action and guiding children's imitation of these actions (see also Southgate et al., 2009). Language can also facilitate the comparison of actions already in 10-month-olds and help them to understand actions as being goal-directed (Gerson and Woodward, 2014).

Thus, the concurrent presentation of information from the word and action domains fosters learning in both domains. The studies reviewed above have all, however, examined the temporal synchrony between (particular) action demonstrations and language input. Here, we extend this to examine the influence of the semantic consistency of concurrently presented action demonstrations on word learning. By semantic consistency we refer to the consistency with which certain actions accompany word-object associations. For example, when parents introduce children to new word-object associations, a hopping action can semantically accompany a rabbit in a communicative interaction, or a snapping action can present a crocodile. Then, these action-word-object triads can either be semantically consistent, namely that the same action accompanies successive presentations of a word-object association (i.e., the rabbit is always hopping), or inconsistent in that different actions accompany each presentation of a word-object association (i.e., the rabbit is sometimes hopping and sometimes snapping).

Indeed, consistency in the input to children appears to have a strong influence on early word learning. Consistent mappings of words and objects are obviously a prerequisite for word learning. This is best observed in cross-situational learning paradigms where learning is based on 12- to 14-month-olds tracking the frequency with which distinct words are presented with distinct objects across successive presentations (e.g., Smith and Yu, 2008). Moreover, studies have shown that consistency in form of the same location associations (Benitez and Smith, 2012; Samuelson et al., 2011), and consistent spatial, temporal and linguistic contexts (Roy et al., 2015) support word learning in early childhood. Also, studies on repetitive story telling find that 3-year-olds learned novel words better when being read the same book (containing the novel words) relative to being read different books with the same novel words (Horst et al., 2011; Williams and Horst, 2014). Finally, demonstrating the benefit of syntactic consistency, Schwab and Lew-Williams (2016) show that consistent sentence structure similarly helps 2-year-olds learn new words. Taken together, consistency plays a crucial role in the acquisition of early word-object mappings, impacting the strength with which such mappings are both formed and later accessed.

However, if more than two aspects in this context co-occur, the intended referents of the label may be ambiguous: for instance, if a particular action demonstration consistently accompanies presentations of a word-object association, the word could either refer to the action or to the object due to their similarly high rates of co-occurrence. If, however, the actions vary while the word and object are consistently presented together, the variability in the action presentation might help in disambiguating the object as the intended referent of the word.

Variability has typically been shown to play a role in structuring abstract knowledge, for example in categorization tasks. For example, variability in visual appearance across trials facilitated learning of perceptual categorization already in 6- to 7-month-olds (Quinn and Bhatt, 2010). Also, 10-month-olds learned to discriminate between a typical and an atypical member of a category, only when the objects in that category varied strongly in their perceptual appearance, 
but not when they were perceptually similar (Mather and Plunkett, 2011). Further, hearing the same words produced by different speakers helped 14-month-olds to discriminate between the previously heard word and a similar sounding word, suggesting that variability in the phonetic detail of speakers may help a child to grasp subtle differences between those words (Rost and McMurray, 2009, 2010). Nonetheless, Twomey et al. (2014) showed that there are limits to the effects of variability on learning: although 30-month-olds would learn labels for categories when the objects varied in color (but not when they were in identical colors), children did not learn category labels when objects varied in shape and color. Thus, too much variability disrupted children's learning of category labels.

But variability does not necessarily disrupt word recognition and can sometimes even help word learning. Junge et al. (2012) presented 9-month-olds with either constant picture-word pairings (i.e., always the same picture of a cat when hearing the label cat), or varying pictures of the same object (i.e., different cats when hearing cat). Both groups showed learning, indicating that neither consistency nor variability influenced their word recognition. Further, Twomey et al. (2017) tested 2-year-olds' word learning abilities while the background colors of the screen either varied in color or stayed consistently white. They found that children only showed target recognition in the variable condition which led them to assume that decontextualization helped the children to form strong word representations. Nonetheless, they also suggest that increased variability might disrupt successful learning and that this might relate to the learning environment: reduced variability might help in rich learning environments and increased background variability in simpler learning environments (see also Radesky and Christakis, 2016; Twomey et al., 2014). Thus, the effect of variability may vary across contexts, and guide attention allocation in different ways, thereby influencing learning behavior (see also Bahrick et al., 2002).

In the present study, we ask whether young children and adults better learn the labels of novel objects when the objects are presented with consistent actions during labeling, i.e., when distinct word-object mappings are consistently accompanied by the same action demonstration, relative to when they are presented with inconsistent actions, i.e., when distinct word-object mappings are accompanied by varying action demonstrations.

We tested three groups of children, and adults as a control group. For the 18-month-olds, we expected that they would learn words for objects, but that their learning would be influenced by the consistency of actions. For the 30-month-olds, being passed the vocabulary spurt, we expected that the consistency of actions does not influence their word learning, and that they would thus learn in both conditions. However, both age groups did not learn in either condition. Therefore, we additionally tested 3- to 4-year-olds, who we expected to learn as they are experienced word learners already and might not be as easily distracted by actions. Therefore, this age group might be most similar to the adults who we expected to learn in both conditions.

\section{2 | METHODS}

\section{1 | Participants}

Fifty-four German monolingual 18-month-olds (range $=17 ; 23$ - 22;55 months; mean $=18 ; 07,25$ girls) and sixty-five 30-month-olds (range $=27 ; 09$ - 32;12 months; mean = 29;72, 30 girls) and fifty-nine 3- to 4-year-olds (range = 38;89 - 47;9 months; mean $=43,44,27$ girls) participated in the experiment. In addition, 60 adults were tested as a control group (range $=19-50$ years; mean $=26 ; 32,38$ women). Twenty-seven additional participants were tested but excluded from the analysis because of unwillingness to participate (2), technical failure (2), calibration issues (4), bilingualism (2), preterm birth (2), impaired hearing (2), familiarity with the presented novel object (2), or insufficient data (11, see Preprocessing for details). Children were recruited from the group's database and participation was rewarded with a book. In order to control for children's language abilities, caregivers of 18- and 30-month-olds completed the German 
FIGURE 1 Blue and yellow toys were used as novel objects. As novel actions, an upward movement with leaning to the sides, and a sideways movement with tilting backwards and forwards were used.

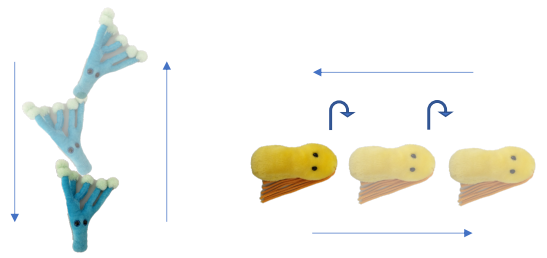

adaption of the FRAKIS (Szagun et al., 2009). To test the language abilities of 3- to 4-year-olds we used the SETK3-5 (Grimm et al., 2010). Adults were mostly students of the University and were rewarded with either 0.5 course credit points or $4 €$. Ethics approval was granted by the University of Göttingen (Project 123).

\section{2 | Stimuli}

We selected two pseudo-words in keeping with German phonotactic constraints (Tanu and Löki), two arbitrary actions (explained in further detail below), and two novel objects (a yellow and a blue soft toy from https://www. giantmicrobes.com/us/, see Fig. 1). The auditory stimuli were recorded by a female German native speaker in infant-directed speech. The labels were embedded in a carrier phrase in both the training phase (e.g., "Schau mal, ein Tanu!", "Look, a Tanu!") and test phase (e.g., "Wo ist denn das Tanu?", "Where is the Tanu?").

The video stimuli consisted of training and test videos. In the training videos, participants saw a hand (with the arm of the agent being visible) moving the objects according to the two selected actions starting from the middle of the screen. We recorded separate videos of both actions being performed on both objects, such that across participants, videos of each distinct action being performed on each object were presented the same number of times. The first action involved a hand moving the object upwards while moving the object side-to-side (from left to right) with each increment in height. It started in the lower middle of the screen, went up and back down again. The second action involved a hand moving the object sideways, while tilting back and forth, moving first to one side of the screen, then back to the other, and ending up in the middle of the screen again. Both actions filled the whole $7 \mathrm{~s}$ of the trial presentation. The auditory stimuli, i.e., the labels for the objects embedded in carrier phrases, were presented at the same time to ensure temporal synchronicity of actions and language (Matatyaho-Bullaro et al., 2014). Each video in the training phase was $7 \mathrm{~s}$ long and $720 \times 420$ pixels in size.

Across the test trials, infants saw the two objects (still images) side-by-side on screen for 5 seconds and were led to fixate the target using carrier phrases (e.g., "Where is the Tanu?") such that the target label began 2.5 s into the video. Pictures in the test phase measured $640 \times 480$ pixels.

\section{3 | Procedure}

Participants sat in a dimly lit and quiet experimental room at a distance of $65 \mathrm{~cm}$ from a TV screen $(92 \times 50 \mathrm{~cm})$. Children sat either in a car seat or on the parent's lap. A remote eye tracker (Tobii X 120), set on a platform underneath the 
FIGURE 2 Display of example setup in the training videos, available on OSF under osf .io/tndj7.

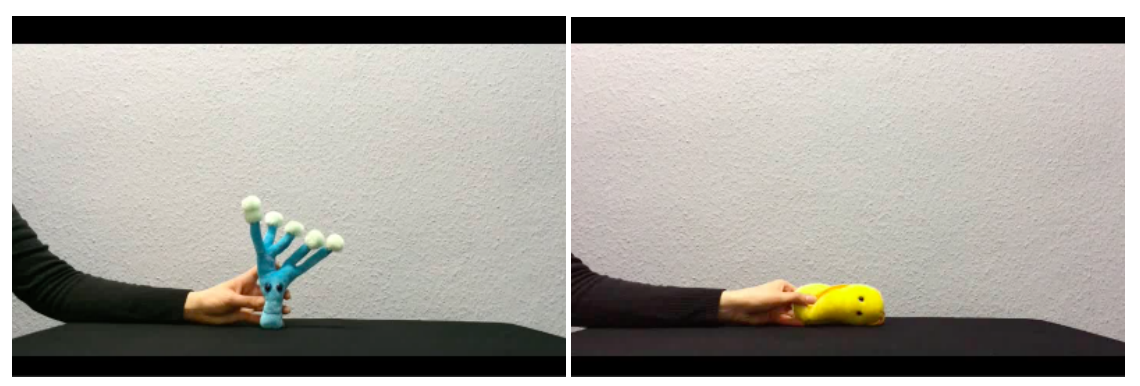

TV screen, was used to record gaze data at $60 \mathrm{~Hz}$. The software E-Prime was used to present the stimuli. Auditory stimuli were presented via two loudspeakers situated above the television screen. Two video cameras centered above the screen served to monitor the participant during the experiment. Calibration was conducted in Tobii Studio using a 5-point calibration procedure, in which a red point appeared in all four corners and the center of the screen. The experiment began following successful calibration. Each trial started with a Teletubby serving as a fixation cross in the middle of the screen against a black background, followed by the stimulus presentation. The videos in the training phase were presented in the center of the screen. Pictures in the test phase appeared next to each other in the center of the left and right half of the screen.

\section{4 | Experimental design}

Each participant was presented with a yoked training and a test phase. The training phase consisted of 4 trials, with each trial presenting the participant with the individual objects in motion accompanied by the label for this object. Participants were presented with 2 trials for each word-object association, and the pairing of objects with labels was counterbalanced across participants. Thus, Tanu was the blue object for half of the participants and the yellow object for the other half. Participants were allocated randomly to one of two conditions. In the Consistent condition, they heard the labels for each individual object while seeing the same action being performed on this object (e.g., the blue object was always presented as moving up, counterbalanced across participants) across both trials. A second group of participants was allocated to the Inconsistent condition where they saw the same objects and heard the same labels. However, here, participants saw both actions being performed on each object across trials (e.g., the blue object was presented as moving up in one trial and moving to the side in a second trial).

The test phase consisted of 8 trials. Each trial presented participants with both images side-by-side on-screen as they heard the label for one of these images exactly half way through the trial, i.e., 2.5 seconds into the trial, dividing it into a pre- and post-naming phase. Gaze points to both images in the pre-naming phase provide an estimate of participants' baseline preference for either of the objects, while gaze points in the post-naming phase indicate participants' response to the relation between the heard label and the images on-screen.

Counterbalancing of novel words, actions, and objects resulted in 6 training lists. Additionally, 4 test lists were created ( 2 words $X 2$ objects, i.e., each object was paired with each label across lists). Presentation order of all trials within blocks was fully randomized. 


\section{5 | Preprocessing}

The eye-tracker provides an estimate of where participants were looking at in each time stamp during the trial, with one data point approximately every $16 \mathrm{~ms}$. All data (gaze data and trial information) were exported from E-Prime and then further processed in R ( $\mathrm{R}$ version 3.2.4 (2016-03-10), R Core Team, 2016). For each time stamp, data were only included when one or both eyes of the participant were tracked reliably (validity less than 2 on E-Prime scale). When both eyes were tracked, the mean gaze point for both eyes was computed for further analysis. Gaze data were then aggregated into $40 \mathrm{~ms}$ bins. Areas of interest were defined according to the size of the displayed objects and their location on screen.

For the test phase, it was coded whether the participant looked at the correct object (i.e., the target), the distractor or at neither of these images on-screen. We analyzed data that began $233 \mathrm{~ms}$ after the onset of the stimulus presentation to ensure that we only analyzed eye movements that could be reliably interpreted as a response (Swingley et al., 1999; Von Holzen and Mani, 2012). Further, we subtracted the mean proportion of target looks of the baseline phase (PTL: total looking time at target/(total looking time at target and distractor)) at each 40 ms time point in the post-naming phase on that particular trial.

Single test trials were excluded if a participant looked at the stimuli less than $M-2 S D$ of the trial duration. Thus, a trial was excluded if an 18-month-old child looked $24.1 \%$ or less of the time during the trial to one of the two pictures. This led to an exclusion of 48 trials (10.5\%). For 30 months, this criterion was at $<=25.7 \%, 62$ trials (11.4\%); for 3 - to 4-year-olds it was at $<=40.6 \%, 40$ trials (9\%); and for adults at $<=37.6 \%, 28$ trials (5.9\%). This led to exclusions of some participants (included above) who did not look enough during any of their trials (18: $n=3 ; 30: n=3 ; 36$ - 48: $n=1$; Adults: $n=1$ ). Furthermore, participants were excluded from the analyses if they provided data from only one trial in the test phase (18: $N=1 ; 30: N=1 ; 36-48: N=1$ ). This left us with 235 participants ( Consistent/n Inconsistent: 18: 28/26; 30: 33/32; 36 - 48: 30/29; Adults: 30/30).

\section{3 | RESULTS}

\section{1 | Description of the growth curve model}

To test these hypotheses, we used Generalised Linear Mixed Models (GLMM) with Time as a factor to model children's looking behaviour during the test phase of the experiment (also coined Growth Curve Analysis (GCA), for a more detailed description and instruction see Mirman et al., 2008; Mirman, 2016). Including time, and its quadratic and cubic polynomial allows us to capture any non-linear change in looking behavior across the whole trial duration (Mirman et al., 2008; Mirman, 2016). Successful learning is assumed to be reflected in the quadratic and cubic polynomials, as their curvature mimics the looking behavior when the participant is looking to the correct object (e.g., an inverted u-shaped curve, i.e., first at chance level, then looking more to the target upon hearing its label, then going back to chance level (quadratic) or lingering above chance level (cubic)). Therefore, we hypothesize that learning in any of the age groups would be reflected on the quadratic or cubic polynomial. If then children learn in one condition but not the other, we expect condition to interact with the respective polynomials which would reflect different curvatures for the two conditions.

We fitted a generalized mixed model using Ime4's Imer function in R (Bates et al., 2015) with Gaussian error structure and identity link function. Condition and Time, and their interaction were included as fixed effects of interest. Further, Label (Tanu, Löki), Object color (blue object, yellow object), Gender, and Age at test were included as control factors. We also included Participant id, Trial number, Label, and Object color as random factors to allow for random 
slopes across participants (but not the correlation parameters among the random intercept and random slopes terms Barr et al. 2013). Trial number and Age at test were z-transformed (to a mean of zero and a standard deviation of one) to simplify later interpretation. A reduced model was fit that did not include Condition. A comparison between the reduced model and the full model including the factor Condition then allows to evaluate the influence of the factor Condition.

We also included Time and its second and third polynomial in the model. This allows us to model the data as a linear, quadratic, and cubic function of time, and thus, to capture according curvatures in the data (the number of polynomials has been restricted to three based on theoretical considerations regarding looking behavior). These variables were also z-transformed (to a mean of zero and a standard deviation of one). An overview of the results can be seen in Tab. 1 and 2 .

Visual inspection of a qq-plot and a histogram of the residuals showed a normal distribution, but homogeneity appeared to be violated based on a plot of residuals against fitted values. Log-transforming the response did not contribute to an improvement of the model. We, therefore, chose the first model, but results need to be reviewed with care.

\section{2 | All age groups}

Comparing the full model with the reduced model revealed that Condition significantly improved the model $(\chi 2=28.55$, $\mathrm{df}=16, p=.027)$. Using drop1, the model revealed a significant interaction of Condition*age* poly3 $(\chi 2=16.4, \mathrm{df}=3, p=$ $.001)$, suggesting differences between age groups and conditions on the linear and cubic term. These results reflect differences in the time course of target recognition between the two conditions across age groups.

To analyze these effects further, we split the data according to the different age groups. To ensure that the participants truly learned the words in both conditions in the age groups, we further split the data according to condition. The results can be seen in Tab. 1 and $2^{1}$.

\section{3 | 18-month-olds}

The model comparison between the full and the reduced model was not significant $(\chi 2=1.71, \mathrm{df}=4, p=.79)$. This suggests that Condition did not interact with 18-month-olds' target recognition.

For neither the model examining the data from the Consistent group alone (Consistent split model), nor the model examining the data from the Inconsistent group alone (Inconsistent split model), did we find significant interactions with the polynomials. Thus, there was no evidence that 18-month-olds had learned and later recognized the word-object mappings in either condition.

\section{4 | 30 -month-olds}

The model comparison between the full and the reduced model was not significant $(\chi 2=4.68, \mathrm{df}=4, p=.32)$. This result suggest that, similar to the 18-month-olds, there was no evidence for differences in 30-month-olds' target recognition based on Condition.

For neither the Consistent split model, nor the Inconsistent split model did we find significant interactions with the polynomials. Thus, there was no evidence that 30-month-olds had learned and later recognized the word-object

\footnotetext{
${ }^{1}$ Running the same models with a subset of the participants who provided information about vocabulary perception, production, and grammar revealed that these factors did not contribute strongly to the models. For comparability across age groups and to preserve a bigger sample size, we focus here on the models without vocabulary information.
} 
mappings in either condition.

\section{5 | 3- to 4-year-olds}

The model comparison between the full and the reduced model was significant $(\chi 2=18.83, \mathrm{df}=4, p<.001)$. Furthermore, there was an interaction between Condition*poly3 $(\chi 2=14.12, \mathrm{df}=1, p<.001)$. These results suggest there was a difference in the pattern of 3- to 4-year-olds' target fixations across the two conditions. Visual inspection revealed that in the Consistent condition, children showed a steep rise in fixating the target shortly after the onset of the target label (from around $500 \mathrm{~ms}$ ) with a peak around $4250 \mathrm{~ms}$. Contrary, looks to the target in the Inconsistent condition hovered at (or just above) chance throughout the trial (see Fig. 3).

For the Consistent split model, the cubic time term was significant $(\chi 2=7.96, \mathrm{df}=1, p=.005)$, suggesting that 3- to 4-year-olds had learned the word-object mappings presented to them in the training phase (in the Consistent condition), reflected in the cubic curvature of their target looking behavior. There was also a tendency for age at test, with an increased overall mean target looking for younger children compared to their older peers $(\chi 2=3.31, \mathrm{df}=1, p=.069)$. This effect arose because their target looking stayed consistently around 1 while the older ones' target looking followed an inverted u-shape curve, which can reduce an overall mean due to the lower start and lower end of the curve. Thus, it seems that learning in this condition tended to be driven by the older 3- to 4-year-olds.

For the Inconsistent split model, we did not find any interactions with the polynomials. Thus, there was no evidence that 3- to 4-year-olds learned the word-object mapping in the Inconsistent condition.

These results highlight the differences in word learning success between the Consistent and Inconsistent condition at 3- to 4-years of age: target recognition followed different trajectories in the two conditions which is reflected in the interaction with the cubic polynomial. The interaction with the linear time term reflects additionally that recognition was overall stronger in the Consistent condition than in the Inconsistent condition. The split models reveal that only in the Consistent condition, did children learn the word-object associations.

\section{6 | Adults}

The model comparison between the full and the reduced model was not significant $(\chi 2=5.81, \mathrm{df}=4, p=.21)$. Nonetheless, there was a trend towards an interaction between Condition*poly2 $(\chi 2=3.36, \mathrm{df}=1, p=.067)$. As the model comparison was not significant, the interactions of Condition and the time polynomial needs to be taken with care.

For the Consistent split model, the cubic time term tended towards significance $(\chi 2=3.35, \mathrm{df}=1, p=.067)$. For the Inconsistent split model, the linear time term was significant $(\chi 2=5.69, \mathrm{df}=1, p=.017)$, and the quadratic time term were significant $(\chi 2=5.52, \mathrm{df}=1, p=.019)$.

These results suggest that adults recognized the target successfully only in the Inconsistent condition. Even for the adults, target recognition followed slightly different trajectories in the two conditions, as can also be seen in Fig. 3 . In the Consistent condition, fixations to the target show a steeper rise and a lower and earlier plateau compared to the Inconsistent condition. Due to the lower plateau, the interaction between the Consistent condition and the cubic term only tended towards significance. 
FIGURE 3 Time course graphs for each age group of participants' baseline-corrected preferential target looking (PTL) during the test phase after label onset at $2500 \mathrm{~ms}$ and $233 \mathrm{~ms}$ to initiate a fixation. The Consistent condition is represented in yellow, the Inconsistent condition is in blue. The line at 0 represents chance level, everything above the line is target looking whereas everything below is distractor looking. The yellow and blue lines reflect the fitted GLMM including Time up to the cubic term for each condition.

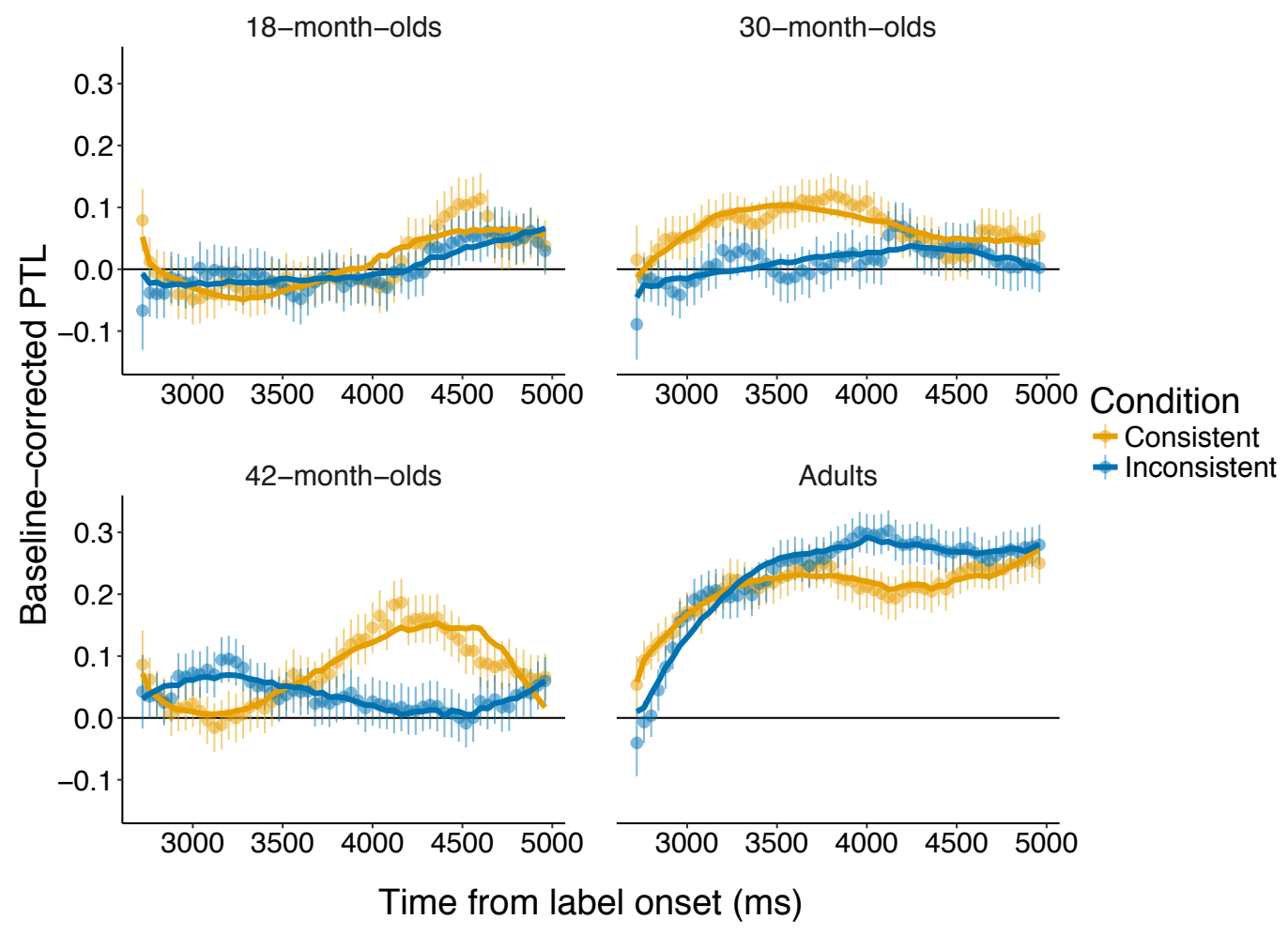

\section{4 | DISCUSSION}

In the current study, we investigated whether the semantic consistency of actions that accompany word-object labellings can influence children's word learning. Participants were presented with novel labels for novel objects while these objects either moved in a consistent (i.e., always the same action across different trials presenting the word-object mapping) or in an inconsistent (i.e., both actions performed on each object across the different trials presenting the word-object mapping) manner. We did not find any evidence that 18-month-olds and 30-month-olds learned the word-object mappings in either condition. In contrast, 3- to 4-year-olds learned the word-object mappings better in the Consistent condition relative to the Inconsistent condition. Indeed, we found evidence that these children learned the word-object mappings only in the Consistent condition. This was reflected in the cubic time term of the GLMM, representing the rise and fall of target looking over time in this condition. Even for the adults, we found differences in the pattern of looks to the target after hearing one of the labels across conditions, with better recognition in the Inconsistent condition.

These results suggest that the semantic consistency of actions influences 3- to 4-year-old children's word learning. 
As Gogate et al. (2000) have shown, actions and words often co-occur in the child's multimodal environment. These actions have also been shown to support word learning (Werker et al., 1998), especially when provided with temporal synchronicity (Matatyaho-Bullaro et al., 2014). However, if these actions appear to be referential, 15-month-olds' word learning is exacerbated (Puccini and Liszkowski, 2012). Our results extend these findings in a critical way, showing that it is not just the temporal consistency with which actions accompany word-object associations that impacts word learning success. At 3- to 4-years of age, children learned the word-object mappings only when each word-object mapping had previously been presented with the same action being performed on the object across separate presentations, i.e., in the consistent condition. This benefit of consistency was not observable at younger ages or with adults. We interpret these findings to suggest that redundant information from different modalities supports the forming of rich lexical representations, but only if this information highlights the word-object association and does not distract from it, and only towards the third year of life.

This is in line with the literature suggesting a beneficial effect of consistency on word learning (Roy et al., 2015), in contrast to a beneficial effect of variability on generalization over different members of a category (Perry et al., 2010; Twomey et al., 2014; Ankowski et al., 2012). Nevertheless, some recent work suggests an impact of lower-level variability (variability in the colour of the background on which objects were presented) on learning of word-object associations (Twomey et al., 2017). In contrast to the beneficial effect of variability in the study by Twomey et al., no such effect was found in our study. We suggest that the function and salience of the additionally varying information might play a crucial role in whether it boosts or detracts attention to word learning. In Twomey et al.'s study, the background colors were a subtle manipulation of the variability in the stimuli. Actions performed on objects, in contrast, may be more salient and compete for attention with the word-object mappings leading to children ignoring the word-object mapping in favor of increased attention to the actions presented. Increased and salient variability can, therefore, disrupt successful learning in a complex learning environment (see Radesky and Christakis, 2016; Twomey et al., 2014). The results of the current study, thus, highlight the importance of a developmental perspective on the influence of cross-domain information on processing. Taken together with the literature on intersensory redundancy, we trace here a developmental pattern in the influence of actions on word learning. Early in life, with actions that do not detract attention from the linguistic input but rather highlight the association between the linguistic input and objects in the world, co-occurring actions bolster word learning. With increasing age, increasing salience (18 and 30 months), and variability in the actions presented (3- to 4-years), actions may indeed deter from word learning and lead to children failing to learn the intended word-object mapping.

Dynamic systems and emergentist approaches to development assume that language acquisition and action processing take place in a complex environment where no single aspect of the environment is a priori responsible for development in any domain (Hirsh-Pasek et al. 2000; Smith and Thelen 1993, see Smith and Thelen, 2003, for an overview). Within this approach, development is the product of active interaction between the child and her immediate environment. As the child develops, she learns to integrate increasing amounts of information while the direct environment adapts to the child's needs and provides the opportunity to learn in a rich and multimodal world.

In their adaptation of such accounts focusing on early word comprehension, Gogate et al. (2001) suggest that word learning results from the interaction of several processes, including selective attention and intersensory perception, as well as the multimodal input provided to the child (based on Sullivan and Horowitz, 1983, and their work on early intermodal perception). Information from different sources and senses regarding the same object might seem arbitrary and irrelevant at first, but, when these information sources co-occur, they help to form a full-blown representation. For example, a word and an object might seem arbitrary when presented independently. But presented in temporal synchrony (Gogate et al., 2000) and potentially even with movement of the object (Gogate and Bahrick, 1998; Werker et al., 1998), the relationship between the word and the object is highlighted for the infant, and therefore supports 
learning. Importantly, perceiving and encoding the relevant aspects of the input requires that the child attends to this input (and indeed, to the relevant aspects of this input). However, attention allocation might be guided by the child's momentary interest in different features of the input. Thus, focusing on a certain type of information can lead to impoverished encoding of other information, which in turn, will shape the spiral staircase of learning in a particular way. Therefore, information from different domains can and does impact processing and learning in the different domains, and can either help or hinder learning across development: Only when the child is able to encode all relevant aspects of the complex multimodal input provided to her, will she be able to avoid such momentary pitfalls of attention allocation. Otherwise, learning can be impeded by the complexity of the input that is beyond the child's resources of attention and perception.

We note, however, that we cannot conclude from the current study that children devoted more attention to the actions relative to the word-object mappings, since children were presented with words and actions simultaneously (Matatyaho and Gogate, 2008). An ongoing study in our lab finds, in keeping with these findings, that younger children (12 and 24 months) do not learn word-object mappings when these are accompanied by actions (see also Puccini and Liszkowski, 2012). Older children (36 months) do learn word-object mappings but not action-object mappings suggesting that at the age where we find an effect of action consistency on word learning, children are able to successfully learn words for objects but not actions for objects. Thus, from a certain age auditory words, and language in particular, may be more salient compared to visually presented actions. And, while being influenced by the consistency of visually presented actions, children are not distracted by these to the extent that they fail to learn words for objects (see Barnhart et al., 2018, for shifts in modality dominance).

Of interest is also the difference between the younger and the older children tested in the current study, for indeed, at this younger age, children failed to learn words altogether in either condition. There was no evidence for target recognition at 18-months and we also did not find evidence for target recognition at 30-months, even though visual inspection of the graph might suggest a tendency towards target recognition in the consistent condition at this later age. One explanation for our failure to find word learning at even this later age is the salience of the co-occurring actions that might detract children from learning the word-world mapping. However, this stands in contrast to previous work with younger infants (Werker et al., 1998) in which even 14-month-olds learned words when these words were presented with objects in motion. In this study, one object (i.e., a dog) was always presented moving to the front of the screen and back while the other (i.e., a toy truck) was presented moving from one side of the screen to the other. Here children only learned the word-object mappings when the objects were presented in motion but not when they were presented without motion,. Notably here, the 14-month-olds were presumably already familiar with images of the dog and the truck and only had to learn the mapping between the water wheel and its label, and it is likely that this increased familiarity with the objects may have led to children mapping the words onto these objects with greater ease (Fennell and Waxman, 2010). The salience of the familiar objects may, therefore, have overridden the effect of salience of the actions on word learning. Furthermore, the children in the present study received only two trials for each object in the training phase. This highly restricted amount of input might have contributed to the low learning rates in the present setting.

In terms of dynamic systems accounts of word learning, our results suggest that the multimodal complexity of the input interacts with the child's abilities to learn. Multimodal input appears to be helpful in certain learning contexts (e.g., Matatyaho and Gogate, 2008), but a child will only learn what is perceptually salient and relevant in his or her contextual and developmental situation at that specific moment (e.g., Gogate et al., 2001). Importantly, we find that the presentation of multimodal input has an impact on word learning, even though this influence is detrimental to word learning at younger ages, especially with salient information from the non-linguistic modality. Our results highlight how children's perception, depending on the age of the child, can be challenged by presenting salient variability in a 
multimodal context (Twomey et al., 2017), and how this can directly influence the spiral staircase of learning: words might not be learned as easily if the child as the active learner is provided with a learning environment that is highly complex, or if that learning environment provides other information that might attract the child's attention. Around age 3 , action consistency seems to have a positive cross-domain influence on word learning. In contrast, adults seem robust against the cross-modal influences between word and action learning. Thus, by adulthood we find limited influence of other domain information on word learning.

In conclusion, we find that children's word learning between 3 and 4 years of age was influenced by the semantic consistency of actions, with consistent word-action-object mappings supporting word learning in contrast to inconsistent word-action-object mappings. Only adults learned words independent of the actions. In terms of a dynamic systems account, this development reflects how the interaction between a developmental stage and the complexity of the multimodal environment plays a role in shaping the learning experience: It shows how we learn to incorporate parts of a rich multimodal environment that learners of any age experience. 
TA B LE 1 GLMM testing differences between conditions on Preferential Target Looking over time including Time, its linear, quadratic, and cubic term. Note: ${ }^{*}=$ not shown because of a very limited interpretation.

res $=\operatorname{Imer}\left(\right.$ PTL_corr.mean Condition*age* ${ }^{*}$ poly1 + poly $2+$ poly 3$)+$ Object + Name + Sex + z.TestAge $+(1+$ poly $1+$ poly $2+$ poly3) || Object:Name $)+(1+$ (poly1 + poly $2+$ poly3) || z.TrialID $)+(1+($ poly1 + poly $2+$ poly3) || id), data=d_aggr, $\mathrm{REML}=\mathrm{F}$, control $=$ contr)

\begin{tabular}{|c|c|c|c|c|c|c|c|c|}
\hline Group & Factor & Estimates & SE & $t$ & $d f$ & AIC & LRT & $p$ \\
\hline \multirow[t]{8}{*}{ Overall } & (Intercept) & -0.033 & 0.053 & -0.618 & $\mathrm{~N}$ & 113173.598 & $\mathrm{~N}$ & $\mathrm{~N}$ \\
\hline & Object & 0.028 & 0.003 & 8.851 & 1 & 113181.621 & 10.022 & 0.002 \\
\hline & Name & 0.013 & 0.003 & 4.173 & 1 & 113178.096 & 6.498 & 0.011 \\
\hline & Sex & 0.003 & 0.025 & 0.134 & 1 & 113171.616 & 0.018 & 0.894 \\
\hline & z.TestAge & -0.044 & 0.123 & -0.359 & 1 & 113171.727 & 0.129 & 0.72 \\
\hline & Condition*Age*poly1 & $*$ & * & * & 3 & 113172.724 & 5.126 & 0.163 \\
\hline & Condition*Age*poly2 & $*$ & * & $*$ & 3 & 113171.002 & 3.404 & 0.333 \\
\hline & Condition*Age*poly3 & $*$ & * & $*$ & 3 & 113183.994 & 16.396 & 0.001 \\
\hline \multirow[t]{8}{*}{18} & (Intercept) & -0.053 & 0.117 & -0.456 & $\mathrm{~N}$ & 24425.557 & $\mathrm{~N}$ & $\mathrm{~N}$ \\
\hline & Object: yellow & 0.042 & 0.007 & 5.874 & 1 & 24431.444 & 7.887 & 0.005 \\
\hline & Name: Tanu & 0.014 & 0.007 & 2.008 & 1 & 24426.961 & 3.403 & 0.065 \\
\hline & Sex: $f$ & -0.062 & 0.038 & -1.656 & 1 & 24426.232 & 2.674 & 0.102 \\
\hline & z.TestAge & -0.214 & 0.384 & -0.557 & 1 & 24423.866 & 0.309 & 0.578 \\
\hline & Condition: Consistent*poly1 & 0.056 & 0.225 & 0.251 & 1 & 24423.62 & 0.063 & 0.802 \\
\hline & Condition: Consistent*poly2 & 0.077 & 0.159 & 0.483 & 1 & 24423.79 & 0.233 & 0.629 \\
\hline & Condition: Consistent*poly3 & -0.122 & 0.111 & -1.098 & 1 & 24424.749 & 1.192 & 0.275 \\
\hline \multirow[t]{8}{*}{30} & (Intercept) & -0.051 & 0.109 & -0.472 & $\mathrm{~N}$ & 33187.624 & $\mathrm{~N}$ & $\mathrm{~N}$ \\
\hline & Object: yellow & -0.017 & 0.007 & -2.602 & 1 & 33190.173 & 4.549 & 0.033 \\
\hline & Name: Tanu & -0.006 & 0.007 & -0.948 & 1 & 33186.522 & 0.898 & 0.343 \\
\hline & Sex: $f$ & 0.024 & 0.035 & 0.704 & 1 & 33186.117 & 0.493 & 0.483 \\
\hline & z.TestAge & 0.174 & 0.23 & 0.756 & 1 & 33186.193 & 0.569 & 0.451 \\
\hline & Condition: Consistent*poly1 & -0.115 & 0.178 & -0.648 & 1 & 33186.042 & 0.418 & 0.518 \\
\hline & Condition: Consistent*poly2 & -0.075 & 0.155 & -0.482 & 1 & 33185.855 & 0.231 & 0.63 \\
\hline & Condition: Consistent*poly3 & 0.153 & 0.104 & 1.469 & 1 & 33187.743 & 2.119 & 0.145 \\
\hline \multirow[t]{8}{*}{$36-48$} & (Intercept) & 0.093 & 0.151 & 0.618 & $\mathrm{~N}$ & 30665.907 & $\mathrm{~N}$ & $\mathrm{~N}$ \\
\hline & Object: yellow & 0.055 & 0.007 & 8.087 & 1 & 30673.242 & 9.335 & 0.002 \\
\hline & Name: Tanu & 0.053 & 0.007 & 7.887 & 1 & 30673.102 & 9.194 & 0.002 \\
\hline & Sex: $f$ & -0.031 & 0.041 & -0.738 & 1 & 30664.451 & 0.544 & 0.461 \\
\hline & z.TestAge & -0.085 & 0.114 & -0.749 & 1 & 30664.466 & 0.559 & 0.455 \\
\hline & Condition: Consistent*poly1 & 0.356 & 0.212 & 1.678 & 1 & 30666.651 & 2.744 & 0.098 \\
\hline & Condition: Consistent*poly2 & -0.13 & 0.184 & -0.707 & 1 & 30664.402 & 0.494 & 0.482 \\
\hline & Condition: Consistent*poly3 & -0.368 & 0.092 & -4.012 & 1 & 30678.023 & 14.115 & $<.001$ \\
\hline \multirow[t]{8}{*}{ Adults } & (Intercept) & 7.438 & 3.574 & 2.081 & $\mathrm{~N}$ & 21923.126 & $\mathrm{~N}$ & $\mathrm{~N}$ \\
\hline & Object: yellow & 0.029 & 0.005 & 5.862 & 1 & 21928.937 & 7.811 & 0.005 \\
\hline & Name: Tanu & 0.001 & 0.005 & 0.224 & 1 & 21921.176 & 0.05 & 0.823 \\
\hline & Sex: $f$ & 0.115 & 0.076 & 1.518 & 1 & 21923.386 & 2.26 & 0.133 \\
\hline & z.TestAge & 5.383 & 2.642 & 2.037 & 1 & 21925.138 & 4.012 & 0.045 \\
\hline & Condition: Consistent*poly1 & -0.247 & 0.178 & -1.393 & 1 & 21923.033 & 1.907 & 0.167 \\
\hline & Condition: Consistent*poly2 & 0.241 & 0.129 & 1.859 & 1 & 21924.485 & 3.359 & 0.067 \\
\hline & Condition: Consistent ${ }^{*}$ poly3 & 0.037 & 0.085 & 0.435 & 1 & 21921.314 & 0.189 & 0.664 \\
\hline
\end{tabular}


TAB LE 2 GLMM testing successful learning within conditions over time including Time, its linear, quadratic, and cubic term. Note: ${ }^{*}=$ not shown because of a very limited interpretation.

res $=\operatorname{Imer}($ PTL_corr.mean (poly1 + poly $2+$ poly 3$)+$ Object + Name + Sex + z.TestAge $+(1+($ poly $1+$ poly $2+$ poly 3$) \|$ Object:Name $)+(1+$ (poly1 + poly2 + poly3) || z.TrialID $)+(1+$ (poly1 + poly $2+$ poly3) || id $)$, data=dadult_Inconsistent, $\mathrm{REML}=\mathrm{F}$, control $=$ contr $)$

\begin{tabular}{|c|c|c|c|c|c|c|c|c|}
\hline Group & Factor & Estimates & SE & $t$ & $d f$ & AIC & LRT & $p$ \\
\hline \multirow[t]{8}{*}{ 18: Consistent } & (Intercept) & 0.004 & 0.261 & 0.017 & $\mathrm{~N}$ & 11696.364 & $\mathrm{~N}$ & $\mathrm{~N}$ \\
\hline & poly1 & 0.326 & 0.271 & 1.204 & 1 & 11695.743 & 1.379 & 0.24 \\
\hline & poly2 & 0.14 & 0.201 & 0.698 & 1 & 11694.843 & 0.48 & 0.489 \\
\hline & poly3 & -0.147 & 0.118 & -1.248 & 1 & 11695.847 & 1.483 & 0.223 \\
\hline & Object: yellow & 0.028 & 0.01 & 2.753 & 1 & 11698.74 & 4.376 & 0.036 \\
\hline & Name: Tanu & 0.059 & 0.01 & 5.874 & 1 & 11701.096 & 6.732 & 0.009 \\
\hline & Sex: $f$ & 0.015 & 0.048 & 0.322 & 1 & 11694.467 & 0.103 & 0.748 \\
\hline & z.TestAge & 0.181 & 0.896 & 0.202 & 1 & 11694.405 & 0.041 & 0.84 \\
\hline \multirow[t]{8}{*}{ 18: Inconsistent } & (Intercept) & 0.042 & 0.134 & 0.317 & $\mathrm{~N}$ & 12335.411 & $\mathrm{~N}$ & $\mathrm{~N}$ \\
\hline & poly1 & 0.211 & 0.209 & 1.01 & 1 & 12334.412 & 1.001 & 0.317 \\
\hline & poly2 & 0.092 & 0.187 & 0.491 & 1 & 12333.65 & 0.239 & 0.625 \\
\hline & poly3 & 0.01 & 0.126 & 0.076 & 1 & 12333.417 & 0.006 & 0.939 \\
\hline & Object: yellow & 0.066 & 0.01 & 6.591 & 1 & 12342.422 & 9.011 & 0.003 \\
\hline & Name: Tanu & -0.021 & 0.01 & -2.158 & 1 & 12337.161 & 3.751 & 0.053 \\
\hline & Sex: $f$ & -0.157 & 0.055 & -2.873 & 1 & 12340.595 & 7.184 & 0.007 \\
\hline & z.TestAge & -0.094 & 0.428 & -0.219 & 1 & 12333.459 & 0.048 & 0.827 \\
\hline \multirow[t]{8}{*}{ 30: Consistent } & (Intercept) & 0.172 & 0.171 & 1.004 & $N$ & 17802.997 & $\mathrm{~N}$ & $\mathrm{~N}$ \\
\hline & poly1 & -0.034 & 0.234 & -0.145 & 1 & 17801.018 & 0.021 & 0.884 \\
\hline & poly2 & -0.165 & 0.216 & -0.763 & 1 & 17801.566 & 0.568 & 0.451 \\
\hline & poly3 & 0.136 & 0.113 & 1.2 & 1 & 17802.358 & 1.36 & 0.243 \\
\hline & Object: yellow & -0.054 & 0.009 & -6.11 & 1 & 17809.185 & 8.187 & 0.004 \\
\hline & Name: Tanu & -0.046 & 0.009 & -5.216 & 1 & 17808.45 & 7.453 & 0.006 \\
\hline & Sex: $f$ & 0.042 & 0.05 & 0.85 & 1 & 17801.711 & 0.714 & 0.398 \\
\hline & z.TestAge & -0.158 & 0.379 & -0.417 & 1 & 17801.171 & 0.174 & 0.677 \\
\hline \multirow[t]{8}{*}{ 30: Inconsistent } & (Intercept) & -0.198 & 0.128 & -1.546 & $N$ & 15106.54 & $\mathrm{~N}$ & $\mathrm{~N}$ \\
\hline & poly1 & 0.118 & 0.202 & 0.586 & 1 & 15104.88 & 0.34 & 0.56 \\
\hline & poly2 & -0.102 & 0.2 & -0.51 & 1 & 15104.797 & 0.257 & 0.612 \\
\hline & poly3 & -0.028 & 0.117 & -0.238 & 1 & 15104.596 & 0.056 & 0.813 \\
\hline & Object: yellow & 0.028 & 0.01 & 2.717 & 1 & 15109.416 & 4.876 & 0.027 \\
\hline & Name: Tanu & 0.039 & 0.01 & 4.013 & 1 & 15111.047 & 6.507 & 0.011 \\
\hline & Sex: $f$ & 0.007 & 0.046 & 0.153 & 1 & 15104.563 & 0.023 & 0.879 \\
\hline & z.TestAge & 0.419 & 0.272 & 1.539 & 1 & 15106.818 & 2.278 & 0.131 \\
\hline \multirow[t]{8}{*}{36 - 48: Consistent } & (Intercept) & 0.357 & 0.194 & 1.839 & $N$ & 15736.594 & $\mathrm{~N}$ & $\mathrm{~N}$ \\
\hline & poly1 & 0.249 & 0.2 & 1.243 & 1 & 15736.087 & 1.493 & 0.222 \\
\hline & poly2 & -0.151 & 0.14 & -1.081 & 1 & 15735.736 & 1.143 & 0.285 \\
\hline & poly3 & -0.241 & 0.075 & -3.219 & 1 & 15742.551 & 7.958 & 0.005 \\
\hline & Object: yellow & 0.058 & 0.01 & 5.926 & 1 & 15743.002 & 8.409 & 0.004 \\
\hline & Name: Tanu & 0.052 & 0.01 & 5.335 & 1 & 15742.446 & 7.852 & 0.005 \\
\hline & Sex: $f$ & 0.037 & 0.053 & 0.697 & 1 & 15735.073 & 0.479 & 0.489 \\
\hline & z.TestAge & -0.274 & 0.147 & -1.861 & 1 & 15737.908 & 3.314 & 0.069 \\
\hline \multirow[t]{2}{*}{36 - 48: Inconsistent } & (Intercept) & -0.086 & 0.222 & -0.389 & $\mathrm{~N}$ & 14700.989 & $\mathrm{~N}$ & $\mathrm{~N}$ \\
\hline & poly1 & -0.121 & 0.238 & -0.507 & 1 & 14699.245 & 0.256 & 0.613 \\
\hline
\end{tabular}




\begin{tabular}{llrrrrrrr} 
Group & Factor & Estimates & SE & $t$ & $d f$ & AIC & LRT & $p$ \\
\hline & poly2 & -0.034 & 0.203 & -0.17 & 1 & 14699.018 & 0.029 & 0.865 \\
& poly3 & 0.125 & 0.142 & 0.879 & 1 & 14699.733 & 0.743 & 0.389 \\
& Object: yellow & 0.061 & 0.01 & 6.376 & 1 & 14706.758 & 7.769 & 0.005 \\
& Name: Tanu & 0.056 & 0.009 & 6.025 & 1 & 14706.47 & 7.481 & 0.006 \\
& Sex:f & -0.079 & 0.06 & -1.318 & 1 & 14700.675 & 1.685 & 0.194 \\
& z.TestAge & 0.07 & 0.166 & 0.421 & 1 & 14699.166 & 0.177 & 0.674 \\
Adults: Consistent & Intercept) & 6.199 & 4.029 & 1.539 & $\mathrm{~N}$ & 11689.973 & $\mathrm{~N}$ & $\mathrm{~N}$ \\
& poly1 & 0.24 & 0.2 & 1.203 & 1 & 11689.381 & 1.408 & 0.235 \\
& poly2 & -0.121 & 0.137 & -0.879 & 1 & 11688.734 & 0.761 & 0.383 \\
& poly3 & 0.159 & 0.076 & 2.1 & 1 & 11691.318 & 3.345 & 0.067 \\
& Object: yellow & 0.044 & 0.007 & 6.42 & 1 & 11696.186 & 8.214 & 0.004 \\
& Name: Tanu & -0.003 & 0.007 & -0.468 & 1 & 11688.192 & 0.219 & 0.64 \\
& Sex:f & 0.106 & 0.104 & 1.018 & 1 & 11688.992 & 1.019 & 0.313 \\
& z.TestAge & 4.497 & 2.995 & 1.501 & 1 & 11690.146 & 2.173 & 0.14 \\
Adults: Inconsistent & Intercept) & 11.482 & 7.47 & 1.537 & $\mathrm{~N}$ & 10058.754 & $\mathrm{~N}$ & $\mathrm{~N}$ \\
& poly1 & 0.479 & 0.166 & 2.875 & 1 & 10062.441 & 5.687 & 0.017 \\
& poly2 & -0.354 & 0.13 & -2.715 & 1 & 10062.269 & 5.515 & 0.019 \\
& poly3 & 0.117 & 0.074 & 1.579 & 1 & 10059.142 & 2.388 & 0.122 \\
& Objectgelb & 0.015 & 0.007 & 2.106 & 1 & 10060.362 & 3.608 & 0.058 \\
& NameTanu & 0.01 & 0.007 & 1.444 & 1 & 10058.837 & 2.083 & 0.149 \\
& Sexw & 0.131 & 0.111 & 1.189 & 1 & 10058.134 & 1.38 & 0.24 \\
& z.TestAge & 8.37 & 5.52 & 1.516 & 1 & 10058.968 & 2.214 & 0.137 \\
\hline
\end{tabular}

\section{ACKNOWLEDGEMENTS}

We thank the families and their children, as well as the adults for participating in the study. Further, we would like to thank Vivien Radtke and Sebastian Isbaner for their helpful comments throughout the process, and Roger Mundry and Dan Mirman for statistical support. Example videos are available via the Open Science Framework under osf . io/tndj7.

\section{REFERENCES}

Ankowski, A. A., Vlach, H. A. and Sandhofer, C. M. (2012) Comparison versus contrast: Task specifics affect category acquisition. Infant and Child Development, 22, 1-23.

Bahrick, L. E., Gogate, L. J. and Ruiz, I. (2002) Attention and memory for faces and actions in infancy: The salience of actions over faces in dynamic events. Child Development, 73, 1629-1643.

Barnhart, W. R., Rivera, S. and Robinson, C. W. (2018) Different patterns of modality dominance across development. Acta Psychologica, 182, 154-165.

Barr, D. J., Levy, R., Scheepers, C. and Tily, H. J. (2013) Random effects structure for confirmatory hypothesis testing: Keep it maximal. Journal of Memory and Language, 68, 255-278.

Bates, D., Mächler, M., Bolker, B. and Walker, S. (2015) Fitting linear mixed-effects models using Ime4. Journal of Statistical Software, 67, 1-48.

Behne, T., Carpenter, M., Call, J. and Tomasello, M. (2005) Unwilling versus unable: infants' understanding of intentional action. Developmental Psychology, 41, 328-337.

Benedict, H. (1979) Early lexical development: Comprehension and production. Journal of Child Language, 6, $183-200$.

Benitez, V. L. and Smith, L. B. (2012) Predictable locations aid early object name learning. Cognition, 125, 339-352. 
Bergelson, E. and Swingley, D. (2012) At 6-9 months, human infants know the meanings of many common nouns. Proceedings of the National Academy of Sciences, 109, 3253-3258.

Brand, R. J. and Tapscott, S. (2007) Acoustic packaging of action sequences by infants. Infancy, 11, 321-332.

Elsner, B. and Pfeifer, C. (2012) Movement or goal: Goal salience and verbal cues affect preschoolers' imitation of action components. Journal of Experimental Child Psychology, 112, 283-295.

Fennell, C. T. and Waxman, S. R. (2010) What paradox? Referential Cues Allow for Infant Use of Phonetic Detail in Word Learning. Child Development, 81, 1376-1383.

Gerson, S. A. and Woodward, A. L. (2014) Labels facilitate infants' comparison of action goals. Journal of Cognition and Development, 15, 197-212.

Gogate, L. J. and Bahrick, L. E. (1998) Intersensory redundancy facilitates learning of arbitrary relations between vowel sounds and objects in seven-month-old infants. Journal of Experimental Child Psychology, 69, 133-149.

Gogate, L. J., Bahrick, L. E. and Watson, J. D. (2000) A study of multimodal motherese: The role of temporal synchrony between verbal labels and gestures. Child Development, 71, 878-894.

Gogate, L. J., Bolzani, L. H. and Betancourt, E. A. (2006) Attention to maternal multimodal naming by 6-to 8-month-old infants and learning of word-object relations. Infancy, 9, 259-288.

Gogate, L. J., Walker-Andrews, A. S. and Bahrick, L. E. (2001) The intersensory origins of word-comprehension: an ecologicaldynamic systems view. Developmental Science, 4, 1-18.

Grimm, H., Aktaş, M. and Frevert, S. (2010) SETK 3-5: Sprachentwicklungstest für drei-bis fünfjährige Kinder (3; 0-5; 11 Jahre); Diagnose von Sprachverarbeitungsfähigkeiten und auditiven Gedächtnisleistungen; Manual. Göttingen: Hogrefe.

Hirsh-Pasek, K., Golinkoff, R. M. and Hollich, G. (2000) An emergentist coalition model for word learning. In Becoming a word learner: A debate on lexical acquisition, 136-164. Oxford University Press Oxford.

Horst, J. S., Parsons, K. L. and Bryan, N. M. (2011) Get the story straight: Contextual repetition promotes word learning from storybooks. Frontiers in Psychology, 2.

Junge, C., Cutler, A. and Hagoort, P. (2012) Electrophysiological evidence of early word learning. Neuropsychologia, 50, 37023712.

Matatyaho, D. J. and Gogate, L. J. (2008) Type of maternal object motion during synchronous naming predicts preverbal infants' learning of word-object relations. Infancy, 13, 172-184.

Matatyaho-Bullaro, D. J., Gogate, L., Mason, Z., Cadavid, S. and Abdel-Mottaleb, M. (2014) Type of object motion facilitates word mapping by preverbal infants. Journal of Experimental Child Psychology, 118, 27-40.

Mather, E. and Plunkett, K. (2011) Same items, different order: Effects of temporal variability on infant categorization. Cognition, 119, 438-447.

Meyer, M., Hard, B., Brand, R. J., McGarvey, M. and Baldwin, D. A. (2011) Acoustic packaging: Maternal speech and action synchrony. IEEE Transactions on Autonomous Mental Development, 3, 154-162.

Mirman, D. (2016) Growth curve analysis and visualization using R. New York: CRC Press.

Mirman, D., Dixon, J. A. and Magnuson, J. S. (2008) Statistical and computational models of the visual world paradigm: Growth curves and individual differences. Journal of Memory and Language, 59, 475-494.

Nicoladis, E., Mayberry, R. I. and Genesee, F. (1999) Gesture and early bilingual development. Developmental Psychology, 35, 514-526. 
Perry, L. K., Samuelson, L. K., Malloy, L. M. and Schiffer, R. N. (2010) Learn locally, think globally: Exemplar variability supports higher-order generalization and word learning. Psychological Science, 21, 1894-1902.

Puccini, D. and Liszkowski, U. (2012) 15-month-old infants fast map words but not representational gestures of multimodal labels. Frontiers in Psychology, 3, 101.

Quinn, P. C. and Bhatt, R. S. (2010) Learning perceptual organization in infancy: The effect of simultaneous versus sequential variability experience. Perception, 39, 795-806.

R Core Team (2016) R: A Language and Environment for Statistical Computing. R Foundation for Statistical Computing, Vienna, Austria. URL: https://www.R-project.org/.

Radesky, J. S. and Christakis, D. A. (2016) Keeping children's attention: The problem with bells and whistles. JAMA pediatrics, 170, 112-113.

Rost, G. C. and McMurray, B. (2009) Speaker variability augments phonological processing in early word learning. Developmental Science, 12, 339-349.

- (2010) Finding the signal by adding noise: The role of noncontrastive phonetic variability in early word learning. Infancy, 15, 608-635.

Roy, B. C., Frank, M. C., DeCamp, P., Miller, M. and Roy, D. (2015) Predicting the birth of a spoken word. Proceedings of the National Academy of Sciences, 112, 12663-12668.

Samuelson, L. K., Smith, L. B., Perry, L. K. and Spencer, J. P. (2011) Grounding word learning in space. PloS One, 6, e28095.

Schwab, J. F. and Lew-Williams, C. (2016) Repetition across successive sentences facilitates young children's word learning. Developmental Psychology, 52, 879-886.

Smith, L. B. and Thelen, E. E. (1993) A dynamic systems approach to development: Applications. In Bradford Books series in cognitive psychology. Cambridge, Massachusetts: The MIT Press.

- (2003) Development as a dynamic system. Trends in Cognitive Sciences, 7, 343-348.

Smith, L. B. and Yu, C. (2008) Infants rapidly learn word-referent mappings via cross-situational statistics. Cognition, 106, 1558-1568.

Sommerville, J. A., Woodward, A. L. and Needham, A. (2005) Action experience alters 3-month-old infants' perception of others' actions. Cognition, 96, B1-B11.

Southgate, V., Chevallier, C. and Csibra, G. (2009) Sensitivity to communicative relevance tells young children what to imitate. Developmental Science, 12, 1013-1019.

Stahl, A. E., Romberg, A. R., Roseberry, S., Golinkoff, R. M. and Hirsh-Pasek, K. (2014) Infants segment continuous events using transitional probabilities. Child Development, 85, 1821-1826.

Sullivan, J. W. and Horowitz, F. D. (1983) Infant intermodal perception and maternal multimodal stimulation: Implications for language development. In Advances in Infancy Research (eds. C. Rovee-Collier and L. Lipsitt), vol. 2, 183-239. Norwood, NJ: Ablex: Elsevier/JAI Press Inc.

Swingley, D., Pinto, J. P. and Fernald, A. (1999) Continuous processing in word recognition at 24 months. Cognition, 71, 73-108.

Szagun, G., Stumper, B. and Schramm, A. S. (2009) Fragebogen zur frühkindlichen Sprachentwicklung (FRAKIS) und FRAKIS-K (Kurzform). Frankfurt/Main, Germany: Pearson Assessment.

Twomey, K. E., Ma, L. and Westermann, G. (2017) All the right noises: Background variability helps early word learning. Cognitive Science, 1-26. 
Twomey, K. E., Ranson, S. L. and Horst, J. S. (2014) That's more like it: Multiple exemplars facilitate word learning. Infant and Child Development, 23, 105-122.

Von Holzen, K. and Mani, N. (2012) Language nonselective lexical access in bilingual toddlers. Journal of Experimental Child Psychology, 113, 569-586.

Werker, J. F., Cohen, L. B., Lloyd, V. L., Casasola, M. and Stager, C. L. (1998) Acquisition of word-object associations by 14month-old infants. Developmental Psychology, 34, 1289-1309.

Williams, S. E. and Horst, J. S. (2014) Goodnight book: sleep consolidation improves word learning via storybooks. Frontiers in Psychology, 5. 